\title{
Annual acknowledgement of reviewers
}

Timothy R Sands

\section{Contributing reviewers}

The editors of BMC Medical Genetics would like to thank all our reviewers who have contributed to the journal in Volume 14 (2013).

\begin{tabular}{|c|c|c|}
\hline $\begin{array}{l}\text { Amir Abbasi } \\
\text { Pakistan }\end{array}$ & $\begin{array}{l}\text { Muhammad Ansar } \\
\text { Pakistan }\end{array}$ & $\begin{array}{l}\text { Sanjay Kumar Banerjee } \\
\text { India }\end{array}$ \\
\hline $\begin{array}{l}\text { Jose Abdenur } \\
\text { USA }\end{array}$ & $\begin{array}{l}\text { Anthony Antonellis } \\
\text { USA }\end{array}$ & $\begin{array}{l}\text { Vladislav Baranov } \\
\text { Russia }\end{array}$ \\
\hline $\begin{array}{l}\text { John Achermann } \\
\text { UK }\end{array}$ & $\begin{array}{l}\text { Paolo Aretini } \\
\text { Italy }\end{array}$ & $\begin{array}{l}\text { Cynthia Bartels } \\
\text { USA }\end{array}$ \\
\hline $\begin{array}{l}\text { Margaret Adam } \\
\text { USA }\end{array}$ & $\begin{array}{l}\text { Tadao Arinami } \\
\text { Japan }\end{array}$ & $\begin{array}{l}\text { Jasmin Bartl } \\
\text { Switzerland }\end{array}$ \\
\hline $\begin{array}{l}\text { Ilir Agalliu } \\
\text { USA }\end{array}$ & $\begin{array}{l}\text { Rector Arya } \\
\text { USA }\end{array}$ & $\begin{array}{l}\text { Daniel Batlle } \\
\text { USA }\end{array}$ \\
\hline $\begin{array}{l}\text { Arvind Ahuja } \\
\text { India }\end{array}$ & $\begin{array}{l}\text { Stella Aslibekyan } \\
\text { USA }\end{array}$ & $\begin{array}{l}\text { Tara Beattie } \\
\text { Canada }\end{array}$ \\
\hline $\begin{array}{l}\text { Masashi Akiyama } \\
\text { Japan }\end{array}$ & $\begin{array}{l}\text { Halil Ibrahim Atasoy } \\
\text { Turkey }\end{array}$ & $\begin{array}{l}\text { Britt Maria Beckmann } \\
\text { Germany }\end{array}$ \\
\hline $\begin{array}{l}\text { Ahmed Al Saleh } \\
\text { Saudi Arabia }\end{array}$ & $\begin{array}{l}\text { Michaela Auer-Grumbach } \\
\text { Austria }\end{array}$ & $\begin{array}{l}\text { Alessandro Beghini } \\
\text { Italy }\end{array}$ \\
\hline $\begin{array}{l}\text { Fowzan Alkuraya } \\
\text { Saudi Arabia }\end{array}$ & $\begin{array}{l}\text { Mona Awadalla } \\
\text { Australia }\end{array}$ & $\begin{array}{l}\text { Elijah Behr } \\
\text { UK }\end{array}$ \\
\hline $\begin{array}{l}\text { Valerie Allamand } \\
\text { France }\end{array}$ & $\begin{array}{l}\text { Hideo Baba } \\
\text { Japan }\end{array}$ & $\begin{array}{l}\text { Tawfeg Ben-Omran } \\
\text { Qatar }\end{array}$ \\
\hline $\begin{array}{l}\text { Seth Alper } \\
\text { USA }\end{array}$ & $\begin{array}{l}\text { Elena Bacchelli } \\
\text { Italy }\end{array}$ & $\begin{array}{l}\text { Conceicao Bettencourt } \\
\text { UK }\end{array}$ \\
\hline $\begin{array}{l}\text { Gregor Andelfinger } \\
\text { Canada }\end{array}$ & $\begin{array}{l}\text { Claudia Bagni } \\
\text { Belgium }\end{array}$ & $\begin{array}{l}\text { Eric Beyer } \\
\text { USA }\end{array}$ \\
\hline $\begin{array}{l}\text { Miguel Andrade-Navarro } \\
\text { Germany }\end{array}$ & $\begin{array}{l}\text { Pantelis Bagos } \\
\text { Greece }\end{array}$ & $\begin{array}{l}\text { Amarjit Bhanwer } \\
\text { India }\end{array}$ \\
\hline $\begin{array}{l}\text { Ivan Angulo } \\
\text { Brazil }\end{array}$ & $\begin{array}{l}\text { Ana Bakija-Konsuo } \\
\text { Croatia }\end{array}$ & $\begin{array}{l}\text { Harald Binder } \\
\text { Germany }\end{array}$ \\
\hline
\end{tabular}

Correspondence: tim.sands@biomedcentral.com

BioMed Central, Floor 6, 236 Gray's Inn Road, London WC1X 8HB, UK 
Kelly Birdwell

USA

Ralf Birkenhäger

Germany

Peyman Björklund

Sweden

Nevado Blanco

Spain

Luigi Boccuto

USA

Jolanda Boer

Netherlands

Carsten Böger

Germany

Anna Rita Bonfigli

Italy

Nabila Bouatia-Naji

France

Anna Boyajyan

Armenia

Bernard Brais

Canada

Francesco Brancati

Italy

Raja Brauner

France

Jerome Brody

USA

Lesley Bruce

UK

Nicola Brunetti-Pierri

Italy

David Bunyan

UK

Adam Butterworth

UK

Carmen Cadilla

USA

Maria A Caligo

Italy

Lisa Cameron

Canada

Federico Canzian

Germany
Nicola Carboni

Jamaica

Melissa Carter

Canada

Sergi Castellvi-Bel

Spain

John Castiblanco

USA

Weichiao Chang

Taiwan

Chia-Hsiang Chen

Taiwan

Ji Yih Chen

Taiwan

Rong Chen

USA

Zhuo Chen

USA

Yu-Ching Cheng

USA

Pietro Chiurazzi

Italy

Murim Choi

USA

Sven Cichon

Switzerland

Marina Colombi

Italy

Lawreen H Connors

USA

Fabio Coppedè

Italy

Marta Cordoba

Argentina

Aiden Corvin

Ireland

Harriet Corvol

France

Gregory Costain

Canada

Frans Cremers

Netherlands

Julio Croda

Brazil
Sergio Cuevas-Covarrubias

Mexico

Hengmi Cui

China

Robert Culverhouse

USA

Francesca Dagna

Italy

Neetu Dahiya

USA

Pu Dai

China

Denise Daley

USA

Bruno Dallapiccola

Italy

Saeed Daneshmandi

Iran

Ignacio Davila

Spain

Camila Andréa De Oliveira

Brazil

Marina De Rosa

Italy

George Dedoussis

Greece

Ignacio del Castillo

Spain

Marcela del Rio

Spain

Ralph Delfino

USA

Jesús Delgado Calle

Spain

Charles DeLisi

USA

Masashi Demura

Japan

Marcella Devoto

USA

Oscar Diaz Horta

USA

Haner Direskeneli

Turkey 


\begin{tabular}{|c|c|c|}
\hline Steven Dobrowolski & Philip Giampietro & Yuji Hiromatsu \\
\hline USA & USA & Japan \\
\hline James Dowling & Michael Gill & Conrad Hodgkinson \\
\hline USA & Ireland & USA \\
\hline Elise Drouin & Hans Goebel & Julia Hoefele \\
\hline USA & Germany & Germany \\
\hline Denis Duboc & Celso Gomez-Sanchez & Linda Holmquist Mengelbier \\
\hline France & USA & Sweden \\
\hline Mathieu Emily & Margarida Gonçalo & Yukio Horikawa \\
\hline France & Portugal & Japan \\
\hline Sean Ennis & Marilda Goncalves & Laura Horsfall \\
\hline Ireland & Brazil & UK \\
\hline Gregory Enns & Miguel Gonzalez-Gay & Chia-Wen Hsu \\
\hline USA & Spain & USA \\
\hline Raul Estevez & Ivan Gorlov & Whady Hueb \\
\hline Spain & USA & Brazil \\
\hline Agustin Fernandez & Grazyna Gromadzka & Maria Hughes \\
\hline Spain & Poland & UK \\
\hline Robert Ferrell & Renzo Guerrini & Maria Hurle \\
\hline USA & Italy & Spain \\
\hline Gianluigi Forloni & Xiong Guo & Mohammad Ilyas \\
\hline Italy & China & UK \\
\hline Renato Franco & Fiorella Gurrieri & Naoko Iwasaki \\
\hline Italy & Italy & Japan \\
\hline Alexis Frazier-Wood & Christiane Haase & David Jeffries \\
\hline USA & Denmark & Gambia \\
\hline Hudson Freeze & Andreas Hadjisavvas & Johan Jocken \\
\hline USA & Cyprus & Netherlands \\
\hline Tomomi Fujisawa & Yaling Han & Jill Johnsen \\
\hline Japan & China & USA \\
\hline John Fyfe & Dana Hancock & Philippe Joly \\
\hline USA & USA & France \\
\hline Xin Gao & Grahame Hardie & Virginia Kaklamani \\
\hline China & UK & USA \\
\hline Valentina Gatta & Muhammad Hassan & Anna Kaminska \\
\hline Italy & Pakistan & Poland \\
\hline Katarzyna Gaweda-Walerych & Michael Hauser & Nahoko Kaniwa \\
\hline Poland & USA & Japan \\
\hline Massimo Gennarelli & Karen Heath & Michael Karampelas \\
\hline Italy & Spain & UK \\
\hline Ivan Gentile & Andreas Hector & Fiona Karet \\
\hline Italy & Germany & UK \\
\hline Maurizio Genuardi & Ken-Ichi Hirano & Norihiro Kato \\
\hline Italy & Japan & Japan \\
\hline
\end{tabular}


Tomohiro Katsuya

Japan

David Kaye

Australia

Chiea Chuen Khor

Singapore

Madhu Khullar

India

Yoshiaki Kikkawa

Japan

Jae-Min Kim

South Korea

Toru Kimura

Japan

Anton Kiselev

Russia

Joakim Klar

Sweden

Michael Klintschar

Germany

Julian Knight

UK

Kevin Knower

Australia

Lester Kobzik

USA

Carsten Korth

Germany

Ian Krantz

USA

Marcin Krawczyk

Germany

Gajanan Kulkarni

Canada

Kung-Kai Kuo

Taiwan

Mateusz Kurzawski

Poland

Wenli Lai

China

Nicoletta Landsberger

Italy

Dominique Langin

France
Helen Latsoudis

Greece

Ben Li

USA

Dar-Shong Lin

Taiwan

Xie Li-Ping

China

Augusto Litonjua

USA

Alberto Loche

Switzerland

Kerstin Ludwig

Germany

Carol MacArthur

USA

Fiona Macdonald

UK

Alastair MacLennan

Australia

Ikuhiro Maeda

Japan

Shiro Maeda

Japan

Shinya Maekawa

Japan

Elena Maestrini

Italy

Nejat Mahdieh

Iran

Anurupa Maitra

India

Shohreh Maleki

Sweden

Giovanni Malerba

Italy

Michelangelo Mancuso

Italy

Valentina Mangano

Italy

Minna Mannikko

Finland

Vanessa Suñé Mattevi

Brazil
Ross McManus

Ireland

Kim McBride

USA

Jacob McCauley

USA

Andrew McIntosh

UK

Carolina Medina-Gomez

Netherlands

Monica Melo

Brazil

Phillip Melton

Australia

Claudia Menzaghi

Italy

Nuala Meyer

USA

Maria Giuseppina Miano

Italy

Anne-Marie Minihane

UK

Prasun Mishra

USA

Takeshi Miyamoto

Japan

Emna Mkaouar-Rebai

Tunisia

Maja Mockenhaupt

Germany

Pooneh Mokarram

Iran

Monika Morak

Germany

Miguel Moreno-Pelayo

Spain

Rodrigo Moreno-Reyes

Belgium

Derek Morris

Ireland

Umberto Moscato

Italy

Bertram Müller-Myhsok

Germany 
Jan-Willem Muntjewerff

Netherlands

\section{Muhammad Naeem}

Pakistan

Masanori Nakagawa

Japan

Koichi Nakanishi

Japan

Esther Nederhof

Netherlands

Giovanni Neri

Italy

Grethe Neumann Andersen

Denmark

Maggie Ng

USA

Vladyslav Nikolayevskyy

UK

Georgios Nikolopoulos

Greece

Cora O'Neill

Ireland

Javier Gonzalo Ocejo-Vinyals

Spain

Bruno Oertel

Germany

Antonio Oliva

Italy

Ken Ong

UK

Alfredo Orrico

Italy

Haruhiko Osawa

Japan

Yuzuru Otsuka

Japan

Cees Oudejans

Netherlands

Mustafa Ozen

Turkey

Sanna Pakkanen

Finland

Barry Palmer

New Zealand
Laura Papi

Italy

Jong Hoon Park

South Korea

Anne Parle-McDermott

Ireland

Eric Pasmant

France

Guglielmina Pepe

Italy

Jose-Luis Perez-Castrillon

Spain

Carolina Perez-Iratxeta

Canada

Tom Pettersson

Finland

Vito Pistoia

Italy

Alessandra Pontillo

Brazil

Ludmila Prokunina-Olsson

USA

Man Qin

China

Jianhua Qiu

China

Sivaramakrishna Rachakonda

Germany

Mangalathu Rajeevan

USA

Michele Ramsay

South Africa

Sajid Rashid

Pakistan

Umbertina Reed

Brazil

Alessandra Renieri

Italy

William Rizzo

USA

Lisa Roberts

South Africa

Sébastien Robiou Du Pont

Canada
Ghislain Rocheleau

Canada

Laia Rodriguez-Revenga

Spain

Angela Rogers

USA

Karen Rouault

France

Victor Ruiz-Perez

Spain

Michael Bjørn Russell

Norway

Malgorzata Rydzanicz

Poland

Khashayar Sakhaee

USA

Towhid Salam

USA

Federica Sangiuolo

Italy

Nobuo Sanjo

Japan

Regie Lyn Santos-Cortez

USA

Richa Saxena

USA

Fernando Scaglia

USA

André Scherag

Germany

Albert Schinzel

Switzerland

Gudrun Schleiermacher

France

Markus Schubert

Germany

John Scott

Australia

Suma Shankar

USA

Makiko Shimizu

Japan

Marwan Shinawi

USA 
Oliver Sieber

Australia

Jean-Pierre Siffroi

France

Dheer Singh

India

Anne Slavotinek

Hugo Sousa

Portugal

Melissa Southey

Australia

Vivi Srivastava

India

Frauke Stanke

Germany

Pawel Stankiewicz

USA

Mouna Stayoussef

Tunisia

Martin Steinberg

USA

Blanka Stiburkova

Czech Republic

Liborio Stuppia

Italy

Masaya Sugiyama

Japan

Pierre Szepetowski

France

Hiroshi Takashima

Japan

Yasuhiro Takeshima

Japan

Tiong Yang Tan

Australia

Muhammad Tariq

USA

Wichittra Tassaneeyakul

Thailand

Alexander Malcolm Taylor

UK

Rob Taylor

UK
Maria Tejero

Mexico

Mustafa Tekin

USA

Jacob Tfelt-Hansen

Denmark

Per Medbøe Thorsby

Norway

Francesco Danilo Tiziano

Italy

Akemi Tomoda

Japan

Léon-Charles Tranchevent

Belgium

Lisbeth Tranebjaerg

Denmark

Daniela Turchetti

Italy

Benjamin Tycko

USA

Ching-Cherng Tzeng

Taiwan

Korkut Ulucan

Turkey

Philippe Vago

France

Marie Van Dijk

Netherlands

Mathilde Varret

France

Cecilia Vecoli

Italy

Patricia Veras

Brazil

Chris Verschoor

Canada

Sebastien Viatte

UK

Alessandra Viel

Italy

Karani Santhanakrishnan

Vimaleswaran

UK

Tatyana Vlaykova

Bulgaria
Venkatasaroja Voruganti

USA

Raimund Wagener

Germany

Tom Walsh

USA

Lei Wan

Taiwan

Hongwei Wang

China

Philine Wangemann

USA

Richard Ward

USA

Nicole Weisschuh

Germany

Bo Wen

China

Marquitta White

USA

Claus Wilke

USA

Nigel Williams

UK

Judy Wong

Canada

Lee-Jun Wong

USA

Bo Xi

China

Sheng Xiang Xiao

China

Xiaolei Xu

USA

Masahito Yamada

Japan

Hidehisa Yamagata

Japan

Kimiko Yamakawa-Kobayashi Japan

Toshiyuki Yamamoto Japan

Shun-Fa Yang

Taiwan 
Shea Ping Yip

China

Koh-Ichiro Yoshiura

Japan

Feng Yue

USA

Tanja Zeller

Germany

Maria-Christina Zennaro

France
Kui Zhang

USA

Hua Zhao

USA

Qi Zhao

USA

Wenlei Zhuo

China

Malek Zihlif

Jordan
Peter Zill

Germany

Elias Zintzaras

Greece

Marcella Zollino

Italy

Gabor Zsurka

Germany

doi:10.1186/1471-2350-15-20

Cite this article as: Sands: Annual

acknowledgement of reviewers. BMC Medical

Genetics 2014 15:20. 